\title{
ESTUDIANTES DE DISEÑO GRÁFICO EN EL ROL DE DESARROLLADORES WEB: UNA EXPERIENCIA DIDÁCTICA CENTRADA EN APRENDIZAJES ACTIVOS
}

\author{
Celia Torres-Blasco \\ Escola d'Art i Superior de Disseny de les Illes Balears, \\ Universitat de les Illes Balears \\ Adolfina Pérez Garcias \\ Universitat de les Illes Balears \\ David García-Solórzano \\ Universitat Oberta de Catalunya
}

RESUMEN: Con la llegada de las nuevas tecnologías, los estudios de Diseño Gráfico -centrados en el desarrollo de capacidades visuales y comunicativashan incorporado disciplinas multimedia e interactivas para hacer frente a los retos laborales. Estas nuevas disciplinas incluyen aspectos competenciales informáticos -necesarios profesionalmente- que se desvían del perfil de estos estudios, provocando dificultades de aprendizaje. Este artículo describe una experiencia docente de diseño, implementación y evaluación llevada a cabo en una asignatura de Estudios Superiores de Diseño Gráfico, donde el alumnado desarrolla webs con los lenguajes HTML y CSS. A través de la Investigación Basada en Diseño se ha verificado que una propuesta fundamentada en el aprendizaje basado en proyectos acompañada por el aprendizaje basado en problemas, la clase invertida y la tecnología educativa, es idónea para este contexto. Las técnicas, instrumentos e indicadores utilizados para evaluar la experiencia han sido: las observaciones de aula, los cuestionarios, la asistencia, la tasa de abandono y las calificaciones. Los resultados han sido positivos, puesto que se ha mejorado la adquisición de competencias, una mayor satisfacción del alumnado, así como mejores resultados académicos.

PALABRAS CLAVE: estrategia de enseñanza-aprendizaje, aprendizaje activo, aprendizaje basado en proyectos, desarrollo web, estudiantes de diseño gráfico.

\section{GRAPHIC DESIGN STUDENTS IN THE ROLE OF WEB DEVELOPERS: A PEDAGOGIC EXPERIENCE BASED ON ACTIVE LEARNING}

\footnotetext{
ABSTRACT: With the advent of new technologies, Graphic Design studies -focused on the development of visual and communication skills- have
} 
incorporated multimedia and interactive disciplines in order to face the industry challenges. These new disciplines include aspects of computer skills -professionally necessary - that deviate from the profile of these studies, causing learning difficulties. This article describes a teaching experience of design, implementation and evaluation carried out in a subject of Bachelor in Graphic Design, where students develop websites built with HTML and CSS. Using DesignBased Research, it has been verified that a proposal based on project-based learning, accompanied by problem-based learning, the flipped classroom and educative technology, is ideal for this context. The techniques, instruments and indicators used to evaluate the experience have been: classroom observations, questionnaires, attendance, dropout rate and grades. The results have been positive, the acquisition of skills has been improved, greater satisfaction has been obtained and better academic results have been achieved.

KEYWORDS: Teaching-learning strategy, activity learning, project-based learning, web development, graphic design students.

Recibido: 26/03/2020

Aceptado: 03/11/2020

Correspondencia: Celia Torres Blasco, Escola d'Art i Superior de Disseny de les Illes Balears, C/ Instituto Balear, $\mathrm{n}^{\mathrm{o}}$ 5, 07012 Palma, Mallorca. Email: ctorres@escoladisseny.com

\section{INTRODUCCIÓN}

Las escuelas de arte y diseño se han centrado principalmente en el desarrollo de las capacidades visuales y comunicativas. Pero con la llegada de las nuevas tecnologías, los currículos han ido adaptando muchas de sus herramientas al uso de la tecnología digital (Amiri, 2011). En el caso de las disciplinas relacionadas con los medios interactivos y multimedia, las tecnologías digitales no son solo herramientas, sino que estas no existirían si no tuvieran ese componente tan importante basado en la tecnología. Y aquí es cuando viene el dilema, el desarrollo de estos productos se está aprendiendo tanto en centros de informática como en las escuelas de arte y diseño, en cada caso bajo la ideología curricular propia. En el ámbito de los estudios de informática, el énfasis se pone en la parte más tecnológica. En el caso contrario están las escuelas de arte y diseño, que se preocupan por la vertiente más artística y comunicativa. Pero la realidad laboral nos muestra que se solicitan, para un mismo puesto, tanto habilidades de diseño como habilidades mínimas para el desarrollo de la interfaz de una web, utilizando el lenguaje de marcado HTML y el lenguaje de hojas de estilo CSS (Dorn and Guzdial, 2010).

Por todo ello, desde las escuelas de arte y diseño es esencial responder a la necesidad de formación tecnológica para que los diseñadores puedan explorar los entornos multimedia e interactivos, y ser capaces de trabajar en colaboración con equipos multidisciplinares. Pero la diferencia más relevante de esta enseñanza-aprendizaje entre los informáticos y los diseñadores reside en el enfoque de esta formación. Tal 
como expone Amiri (2011), el diseñador solo llegará a ser un "turista de la programación", y por lo tanto, se tendrá que ofrecer una metodología alternativa adecuada a este contexto que facilite el aprendizaje de estas competencias.

En este artículo se describe una experiencia docente de diseño, implementación y evaluación llevada a cabo en una asignatura de Diseño Gráfico de los Estudios Superiores de Diseño de las Islas Baleares, donde el alumnado tiene dificultades a la hora de adquirir las competencias para el desarrollo web a través de HTML y CSS, por ser una materia que se desvía del perfil de estos estudios. Esta experiencia se ha llevado a cabo a lo largo de los cursos 15-16, 16-17 y 17-18 donde han participado un total de 67 estudiantes. Previamente, se realizó una investigación (Torres Blasco, 2016) en los cursos 12-13, 13-14 y 14-15 donde la reflexión sobre el aprendizaje, la satisfacción, confianza y motivación del alumnado, los resultados académicos y la tasa de abandono animó a plantear un cambio metodológico incorporando metodologías activas basadas en el aprendizaje basado en proyectos (ABPr), el aprendizaje basado en problemas (ABP) y la clase invertida con la ayuda de la tecnología educativa (TE).

Tal como expone Imaz (2015), las metodologías activas como ABPr están dado resultados positivos en la universidades, ya que aumentan la motivación del alumnado, disminuyen la tasa de abandono, ayudan a adquirir competencias profesionales y facilitan la interrelación de conocimientos de materias diferentes. Según Gallego Carrillo et al. (2016), el ABPr, el ABP y la clase invertida son una buena combinación, ya que con el modelo pedagógico de la clase invertida se transfieren determinados procesos de enseñanza-aprendizaje fuera del aula para trabajar procesos complejos de forma presencial, en este caso con el ABPr y el ABP. Por otra parte, Lázaro Cayuso (2017) apuesta por una integración tecnológica y propone en su artículo el ABPr como la base y unión de diferentes estrategias como la clase invertida y el trabajo colaborativo. Así mismo, según el Informe Horizon 2019 (Alexander et al., 2019), la TE aporta flexibilidad y personalización, rompiendo así el desafío que supone la brecha de logros en la Enseñanza Superior. Un ejemplo son los recursos educativos abiertos (REA), que son atractivas plataformas en línea que ayudan a los estudiantes a adquirir competencias más consistentes y que ayudan a poner en práctica el modelo de clase invertida.

\section{ANÁlISIS DE LA PROPUESTA DIDÁCTICA PREVIA A ESTA EXPERIENCIA DOCENTE}

La propuesta didáctica aplicada en los cursos 12-13, 13-14 y 14-15 (Torres Blasco, 2016) estaba conformada por los siguientes componentes:

- El modelo pedagógico tradicional expositivo-demostrativo donde la docente explicaba los contenidos presencialmente y los alumnos ejercían un rol pasivo. Era el elemento que tenía más peso y entorno al que giraban el resto de componentes.

- El método didáctico ABP, basado en un proceso de indagación del estudiante para resolver preguntas, donde el alumnado interpretaba y aplicaba la información explicada en clase. Estos problemas se trabajaban tanto en clase como en casa y se resolvían por parte de la docente de una forma demostrativa presencial.

- El método didáctico ABPr, organizado entorno a un proyecto con participación activa del estudiante, donde se trabajaba de manera individual en un proyecto web a final de curso realizado en casa mayoritariamente. 
Esta propuesta fue analizada recopilando la siguiente información:

- A través de las entrevistas grupales y de las observaciones de aula se extrajo el grado de satisfacción del alumnado y cómo se había desarrollado la asignatura. Para los estudiantes la asignatura era de gran complejidad y daban mucha importancia a la asistencia para poder seguir la asignatura y reducir el riesgo de abandono. En clase se sentían inseguros y se desmotivaban. Sugirieron reducir clases teóricas y practicar más en clase para ser supervisados por la docente. Además, propusieron resolver ellos mismos las tareas delante del resto de estudiantes.

- La tasa de abandono ${ }^{1}$ fue uno de los aspectos que más sorprendieron si lo comparamos con la tasa media de la especialidad en esos mismos tres cursos, la cual estaba situada en el $14.5 \%$. En nuestro caso, el promedio era del $21.7 \%$ y llego a ascender al $40 \%$.

- Las calificaciones de los estudiantes que no abandonaban la asignatura eran, en su gran mayoría positivas, ya que solo un $4.3 \%$ suspendían y el $56.5 \%$ obtuvieron 7 o más de calificación.

- En cuanto a la asistencia, el $65.2 \%$ asistía regularmente a clase ( $\geq 70 \%$ de las clases, que es el porcentaje que la guía docente marca para poder ser evaluado en evaluación continua en vez de en evaluación final), el 13\% de estudiantes asistía entre $50 \%$ y $69 \%$ de las clases y el $21.7 \%$ asistía a menos del $50 \%$ de las clases.

A modo de resumen, en la tabla 1 se pueden ver las características de esta experiencia previa.

Tabla 1. Características de la propuesta didáctica previa.

\begin{tabular}{|c|c|c|c|c|}
\hline & Curso & Componentes didácticos & $\begin{array}{l}\text { Técnicas e instrumentos / } \\
\text { indicadores }\end{array}$ & Resultados \\
\hline \multirow{3}{*}{$\begin{array}{l}\text { Propuesta } \\
\text { didáctica } \\
\text { previa } \\
\text { (Torres } \\
\text { Blasco, } \\
\text { 2016) }\end{array}$} & $12-13$ & \multirow{3}{*}{$\begin{array}{l}\text { - Modelo pedagógico } \\
\text { expositivo demostrativo } \\
\text { - ABPr } \\
\text { - ABP }\end{array}$} & \multirow{3}{*}{$\begin{aligned} \text { - } & \text { Cuestionario inicial } \\
& \text { (perfil alumnado y nivel } \\
& \text { de conocimientos) } \\
\text { - } & \text { Observaciones de aula } \\
\text { - } & \text { Entrevistas en grupo } \\
& \text { (satisfacción estudiantes) } \\
\text { - } & \text { Calificaciones } \\
\text { - } & \text { Asistencia } \\
\text { - } & \text { Tasa de abandono }\end{aligned}$} & \multirow{3}{*}{$\begin{array}{l}\text { - Gran complejidad de } \\
\text { la asignatura } \\
\text { - Excesivas clases } \\
\text { teóricas } \\
\text { - Inseguridad y } \\
\text { desmotivación } \\
\text { - } \text { Alta tasa de } \\
\text { abandono } \\
\text { - Buena asistencia } \\
\text { - } \text { Calificaciones } \\
\text { mejorables }\end{array}$} \\
\hline & $13-14$ & & & \\
\hline & $14-15$ & & & \\
\hline
\end{tabular}

De esta forma, se llegó a la conclusión de que era una asignatura compleja y que había que captar la atención del alumnado desde el primer momento, transmitirles confianza competencial y apoyarlos con un mayor acompañamiento. Así pues, se definieron una serie de principios que tenía que cumplir la nueva propuesta didáctica:

1. En este trabajo los abandonos son la suma de estudiantes que se dan de baja, son baja por impago o documentación incorrecta, y los que no se presentan. 
- Acompañar en el aprendizaje dedicando más tiempo en clase para el desarrollo de tareas prácticas y la supervisión.

- Potenciar el aprendizaje significativo con más tiempo para problemas y proyectos.

- Resolución de ejercicios por parte de los alumnos delante del resto de la clase para aprender tanto del proceso como de los aciertos y errores.

- Conseguir una mayor interiorización de los conocimientos con práctica continua, una progresión suave del nivel de dificultad de tareas y mayor flexibilidad en el proceso de aprendizaje.

- Captar la atención con estrategias variadas y materiales multimedia.

- Crear un aula virtual para la comunicación fuera del aula, que organice mejor los recursos de apoyo, los ejercicios y la realización de las entregas.

\section{Método}

\subsection{Problema de investigación}

En una sola asignatura de Diseño Gráfico el alumnado debe adquirir las competencias para el desarrollo web a través de los lenguajes HTML y CSS. Durante el curso, los alumnos manifiestan la gran complejidad de esta disciplina y las dificultades para afrontarla debido a que es una materia que, aunque es necesaria para completar su formación de cara al futuro profesional, se aleja del perfil de estos estudios. Así pues, se quiere reflexionar sobre si un cambio pedagógico basado en aprendizajes activos como el ABPr, el ABP, la clase invertida y acompañado por la TE, es idóneo y mejoraría la satisfacción del alumnado en la materia, la adquisición de competencias y un mejor rendimiento académico.

\subsection{Objetivo}

El objetivo general de esta investigación es diseñar, implementar y evaluar una propuesta de enseñanza-aprendizaje que facilite la adquisición de competencias relacionadas con el desarrollo web con HTML y CSS por parte de estudiantes de Diseño Gráfico en los Estudios Superiores de Diseño, quienes tienen dificultades de aprendizaje por ser una materia que se aleja de su zona de confort, y los prepare para su futuro profesional.

\subsection{Metodología}

El tipo de investigación que se ha aplicado es la Investigación Basada en Diseño (IBD), donde docencia e investigación están estrechamente conectadas y se retroalimentan para mejorar la práctica educativa. Tal como exponen De Benito Crosetti y Salinas Ibáñez (2016), la IBD surge de la necesidad de dar respuesta a los problemas que acontecen en el día a día de la realidad educativa a través de la investigación como proceso transformador.

La estrategia IBD se basa en un proceso cíclico iterativo, compuesto según De Benito Crosetti y Salinas Ibáñez (2016), de las siguientes fases: 
1. La fase de concienciación donde se identifican la naturaleza del problema y los factores implicados, se contrasta con la literatura publicada y se elabora una teoría de trabajo para cambiar la situación.

2. La fase de ideación donde se diseñan las acciones que deberían resolver o reducir el problema.

3. La fase de desarrollo en la cual se implementa el diseño y se adoptan las medidas según el plan previsto.

4. La fase de evaluación en la cual se establece si los planes se llevaron a cabo, y si resolvieron o atenuaron el problema, y en definitiva si se desviaron o no de las expectativas.

5. La fase de conclusión donde se comprueba aquello que se ha logrado en términos de resultados prácticos y nuevos conocimientos. También se analiza si se requiere un nuevo ciclo para solventar problemas o corregir resultados inesperados.

\subsection{Técnicas e instrumentos}

Para evaluar si un cambio metodológico resuelve el problema de investigación:

- Valoraremos si las propuestas didácticas son adecuadas a nuestro contexto a través de observaciones de aula.

- Evaluaremos la satisfacción de los estudiantes mediante un cuestionario.

- Reflexionaremos sobre el seguimiento de la asignatura a través de la asistencia y la tasa de abandono de la experiencia previa y la actual.

- Valoraremos el rendimiento académico por medio de las calificaciones finales de la experiencia previa y la actual.

En cuanto a la gestión de datos, se les pasó al alumnado un consentimiento informado donde se explicaba en qué consistía su participación, el carácter voluntario de participación y la opción de volverse atrás, la confidencialidad y guarda segura de datos, y la anonimización de los informes.

A continuación, se detallan las técnicas e instrumentos utilizados:

\section{Cuestionario inicial}

Con el objetivo de conocer el perfil del alumnado y sus conocimientos sobre la materia, en cada curso se realizó un cuestionario al comienzo de la asignatura. En un primer bloque se les preguntaba sobre el tipo de asignaturas que se les daba mejor según las tres modalidades de bachillerato que ofrece el Ministerio de Educación y Formación Profesional. El segundo bloque, para saber los conocimientos previos sobre la asignatura, estaba formado por preguntas abiertas elaboradas a partir de los contenidos de la asignatura. Por ejemplo, ¿qué es y qué funcionalidad tiene el lenguaje HTML y CSS? El cuestionario se realizó presencialmente en clase y se rellenó on-line para obtener datos automatizados. 


\section{Observaciones de aula}

Debido a que la profesora encargada de la asignatura, era la persona que realizó las observaciones de aula cada curso, se puede decir que se ha realizado una observación directa y participante, cercana a un diario de enseñanza de la docente (Gutiérrez Quintana, 2007). Estas observaciones no estaban estructuradas y las anotaciones se apuntaban tan pronto como era posible o al final de la clase. En ellas se anotaban incidencias, avance de las actividades, dudas, preocupaciones, quejas, motivaciones, comunicación no verbal, conversaciones informales y participación. El objetivo era recopilar, de una forma no invasiva, información para analizar posteriormente la idoneidad de la propuesta didáctica. Además, las observaciones de aula nos ayudarán a contrastar la información del cuestionario de satisfacción, el seguimiento de la asignatura y los resultados académicos.

\section{Cuestionario de satisfacción}

Cada curso se realizó de forma anónima, en clase y al final de la asignatura un cuestionario on-line para sistematizar la recogida de datos estandarizados. No se optó por entrevistas individuales por no sobrecargar al alumno a final de semestre. El cuestionario se desarrolló con el fin de recopilar información concreta que respondiera a las necesidades del estudio planteado. Se tomó como punto de partida el cuestionario de satisfacción de las asignaturas del grado de la Universitat Autònoma de Barcelona $(\mathrm{UAB})$ (s. f.) y el cuestionario de satisfacción del estudiante con la docencia de la Universidad Pública de Navarra (UPNA) (s. f.). Ambos cuestionarios se han desarrollado, implementado y revisado dentro del Sistema de Garantía Interna de Calidad acreditado de las respectivas universidades con el objetivo de realizar un estudio sobre la satisfacción de los estudiantes con la actividad docente de todas las asignaturas y hacer propuestas de mejora. Nuestro objetivo sobre el cuestionario era evaluar el grado de satisfacción del alumnado sobre el funcionamiento de la asignatura, recoger sus opiniones sobre el proceso de su aprendizaje y sugerencias de mejora. Las preguntas estaban divididas en 3 bloques. Había preguntas en escala Likert de 5 puntos para no forzar la elección y evitar inducir un error en los datos (Matas, 2018)-donde 5 era mucho y 1 poco-, preguntas con alternativas múltiples y preguntas abiertas:

- El bloque 1 incluía 2 preguntas de autoevaluación del alumnado en escala Likert sobre cómo se veía respecto a la asignatura. La primera se ha añadido nueva respecto a los cuestionarios de la UPNA y UAB, y la segunda se ha transformado a partir de la pregunta I de la UPNA y la 2 y 3 de la UAB.

- El bloque 2 estaba formado por 16 preguntas de valoración y opinión de la asignatura, 15 en escala Likert y 1 con alternativas múltiples. Las preguntas 1 , $2,3,7,8,10,11$ y 12 han sido formuladas a partir de las preguntas 1, 3, 4 de la UPNA, y de la 5, 6, 7 y 9 de la UAB. La 4, 5, 6, 9, 13, 14, 15 y la 16 están directamente relacionadas con esta experiencia y se han redactado ad hoc.

- El bloque 3 incluía 4 preguntas abiertas para realizar sugerencias sobre los puntos fuertes y débiles de la asignatura, así como los aspectos que mejorarían. Las preguntas 1, 2 y 4 se han formulado a partir de la pregunta 11 y 12 de 
la UPNA y de la 10 y 11 de la UAB. En cambio, la pregunta 3 se ha añadido nueva al cuestionario.

\subsection{Contexto}

La experiencia se ha desarrollado sobre una asignatura de 6 ECTS que se realiza en el primer semestre de $3^{\circ}$ de Diseño Gráfico de los Estudios Superiores de Diseño de las Islas Baleares. El total de estudiantes que han participado son 67, de edades comprendidas entre los 21 años hasta los 35, de los cuales 36 eran mujeres y 31 hombres.

De acuerdo con el cuestionario inicial, el 70.6\% del alumnado aseguró que se le dan mejor las asignaturas relacionadas con la modalidad de Artes, que son un tipo de asignaturas mayoritarias en los estudios de Diseño Gráfico, un 23.5\% eligió las asignaturas de Ciencias y un 5.9\% las asignaturas de Humanidades y Ciencias Sociales. Además, según las respuestas sobre conocimientos de la asignatura del cuestionario inicial, el alumnado tenía conocimientos generales como usuario de Internet, pero no tenían conocimientos previos relevantes como para obviar la parte introductoria de la asignatura.

\section{Diseño de la nueva propuesta didáctica}

La nueva propuesta de enseñanza-aprendizaje para los cursos 15-16, 16-17 y 17-18 estaba centrada en el estudiante, donde el foco principal se colocaba en los aprendizajes activos como el ABPr. Debido a la dificultad que supone esta asignatura para los estudiantes, y que estos no tienen conocimientos previos sobre la materia, antes de abordar el ABPr era necesario construir un camino previo -adaptado a las diferentes capacidades y ritmos de aprendizaje- mediante el cual los estudiantes pudieran adquirir unas competencias mínimas. Así pues, se trabajó con los juegos serios gamificados y el ABP. Para dedicar más tiempo en clase al ABPr y ABP, se disminuyeron un $30 \%$ las clases expositivas-demostrativas y se introdujo la clase invertida con la ayuda de los REA (véase Fig. 1).

Figura 1. Distribución de las técnicas didácticas de la presente propuesta

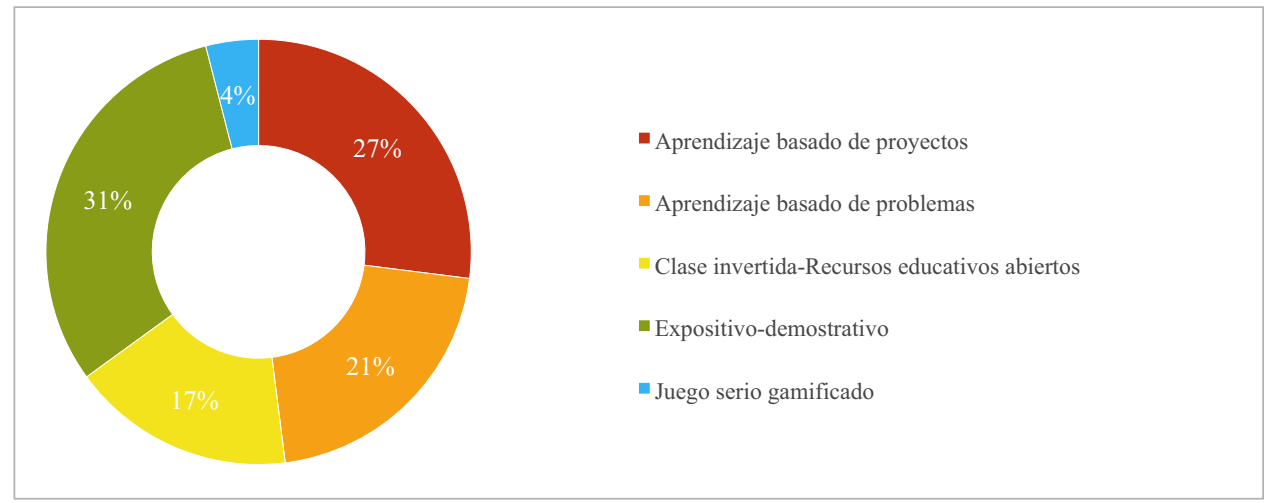


A continuación, se detalla de forma más amplia la propuesta:

- Para introducir el HTML, sus etiquetas y su sintaxis, se ha utilizado el juego serio de cartas cHTeMeLe ${ }^{2}$ con licencia Creative Commons. Los juegos serios tienen objetivos concretos de aprendizaje donde el error se naturaliza y se neutraliza gracias a la respuesta inmediata de sus participantes. Se basan en el aprender haciendo, consiguiendo un aprendizaje profundo y duradero (Gallego-Durán et al., 2014).

- En cuanto al ABP, se han realizado ejercicios cortos sobre los contenidos de la asignatura; por ejemplo, la realización de una hoja de estilos con las etiquetas HTML principales. Respecto a la experiencia previa, se ha aumentado el tiempo en clase para esta práctica, se han definido mejor los objetivos de cada ejercicio y se ha conseguido una continuidad más homogénea entre ellos. Además, las soluciones han sido expuestas por los alumnos en clase, ya que analizar problemas resueltos es una demostración del buen hacer, tanto del resultado final como del proceso a seguir (Campaña et al., 2016). De esta manera, se ha garantizado la participación, cooperación, la responsabilidad, el interés y una base más sólida para afrontar el proyecto final.

- Para el ABPr se ha propuesto como proyecto individual el desarrollo de una web de 2 páginas. En una primera fase se realizaba: la descripción del contenido, el mapa de navegación, el diseño de mockups con sus versiones responsive y el planteamiento de la estructura de etiquetas HTML y los estilos CSS. En la segunda fase se implementaba la web. En total se destinaron 7 sesiones presenciales -con una dedicación de 16 horas- y 24 horas no presenciales de trabajo autónomo del alumnado. Se realizó una presentación en clase en la tercera sesión, en un tiempo máximo de 3 minutos, donde se mostraba el trabajo realizado en la primera fase. In situ se hizo una retroalimentación por parte de la docente y los compañeros. Posteriormente, después de una revisión exhaustiva, la docente les pasó anotaciones con mejoras. Durante el resto de sesiones, al inicio de la clase se presentaban las dudas y se resolvían en grupo o de forma individual según el caso. En la entrega final se hizo una presentación funcional del proyecto para ver todos los resultados. La diferencia con la propuesta anterior reside en el aumento de protagonismo del alumnado. Para ello, se han destinado más horas de trabajo en clase, se ha promovido la resolución conjunta de dudas y el apoyo entre compañeros, y se ha aumentado la supervisión de la docente para conseguir un acompañamiento de calidad.

Con el ABPr, el estudiante es consciente y responsable de su aprendizaje, donde lo importante es tanto el resultado como el proceso (Sosa Sánchez-Cortés et al., 2005). Estos autores consideran el aprendizaje constructivista intrínseco a las disciplinas del área informática, ya que se buscan soluciones a un problema o realidad simulada resuelta a través de un enfoque centrado en el estudiante con un proceso de aprender haciendo. Según Botella Nicolás y Ramos Ramos (2019), el ABPr es un proceso activo que favorece la autonomía del alumno, fomenta su relación con el entorno social y potencia su sentimiento de competencia.

2. http://www.lagrottedubarbu.com/2012/09/11/chtemele-le-jeu-de-plateau-certifie-html5/ 
- Para dedicar más tiempo al ABPr y ABP, el modelo expositivo-demostrativo se ha reducido del $61 \%$ al $31 \%$. Por ser un modelo rápido y eficaz (Luján Mora and Saquete Boró, 2017), se ha utilizado para impartir contenidos introductorios facilitando así su ordenación, así como para impartir contenidos complejos. Sin embargo, las clases demostrativas se han mejorado respecto a cursos anteriores gracias al recurso en línea w3schools ${ }^{3}$. Así, mientras la docente explicaba, los alumnos podían poner en práctica y comprobar al mismo tiempo sus conocimientos.

- Para reducir las clases expositivas-demostrativas sin perder el volumen y complejidad de los contenidos, se ha utilizado la clase invertida. De este modo, los estudiantes se preparaban contenido sencillo en casa y a su ritmo, ganando tiempo para las clases prácticas -que son tareas que requieren mayor interacción con los compañeros- y el asesoramiento personalizado del profesorado. La clase invertida posiciona a los estudiantes en un lugar activo en relación a su proceso de aprendizaje. Es un modelo que ha dado buenos resultados, como es el caso de las investigaciones de Sánchez Rodríguez et al. (2014), donde el alumnado prefería las clases invertidas frente a las clases magistrales por su eficacia. Para la puesta en práctica de la clase invertida, se ha utilizado la TE como los REA en línea Codecademy ${ }^{4}$ y Khanacademy ${ }^{5}$ para el autoaprendizaje de HTML y CSS. Estos recursos facilitan la autoevaluación, ya que ofrecen una visualización directa del resultado, y no dejan pasar de ejercicio si persisten los errores en el ejercicio actual. Según el artículo de Olsson y Mozelius (2016), Codecademy es uno de los entornos preferidos por el alumnado por su utilidad y facilidad de uso. En cuanto a Khanacademy, introduce vídeos cuyas explicaciones agilizan el proceso de aprendizaje (Venter, 2020). Estos recursos ayudan a sistematizar la sintaxis de HTML y CSS liberando carga memorística al cerebro.

- La organización de la asignatura se ha realizado con el aula virtual Classroom ${ }^{6}$, cuyas ventajas son la organización de los materiales, la comunicación fluida entre el grupo fuera del aula, la entrega en línea de tareas, la visualización individual de notas y rúbricas, y la devolución de tareas con correcciones.

\section{Resultados}

Una vez implementada la nueva propuesta didáctica se ha evaluado el diseño realizado mediante: la información recogida de las observaciones de aula y del cuestionario de satisfacción, la asistencia, la tasa de abandono y las calificaciones.

\subsection{Observaciones de aula}

Toda la información recopilada en las observaciones de aula -del presente estudio- fueron codificadas por temas. Se detectó que los estudiantes que no asistían

\footnotetext{
3. https://www.w3schools.com

4. http://www.codecademy.com/

5. https://es.khanacademy.org

6. https://classroom.google.com/
} 
a clase o no estaban atentos en las primeras sesiones se perdían enseguida, se desmotivaban y tenían problemas para continuar. Además, solían quejarse del volumen de trabajo y la dificultad de los contenidos. En cambio, a aquellos que sí asistían, se les veía atentos y motivados cuando se trabajaba con variedad de recursos como Codecademy y Khanacademy. Además, con el juego cHTeMeLe se divirtieron y les ayudó a entender la sintaxis del HMTL. Con el ABP el alumnado se sentía cómodo y seguro. El hecho de realizar las correcciones de los problemas en clase por parte de los estudiantes fue muy positivo, tanto por visualizar ejemplos de buenas prácticas, como por ver errores comunes. En el caso del ABPr, al principio los estudiantes estaban un poco inseguros, pero el aumento de clases presenciales dedicadas al proyecto promovió mayor interacción entre compañeros y más supervisión por parte de la profesora, lo que provocó enseguida un aumento de la seguridad y de la motivación.

\subsection{Cuestionario de satisfacción}

El cuestionario de satisfacción tuvo gran éxito de participación, puesto que fue contestado por 51 estudiantes de los 67 matriculados, que equivale al $76.1 \%$ del total del alumnado matriculado en los tres cursos. A continuación, podemos ver el resultado de los diferentes bloques de preguntas.

\section{Bloque 1: autoevaluación del alumnado}

La autoevaluación de los estudiantes sobre el grado de implicación en la asignatura refleja que el $70.6 \%$ se ha implicado entre el nivel 4 y 5 de la escala Likert, donde 5 es el nivel más alto (véase Fig. 2). Por otra parte, el $47 \%$ del alumnado afirma su alta seguridad en la materia entre el nivel 4 y 5 . En cambio, el $39.2 \%$ se posiciona en el punto 3 de la escala.

Figura 2. Autoevaluación del alumnado

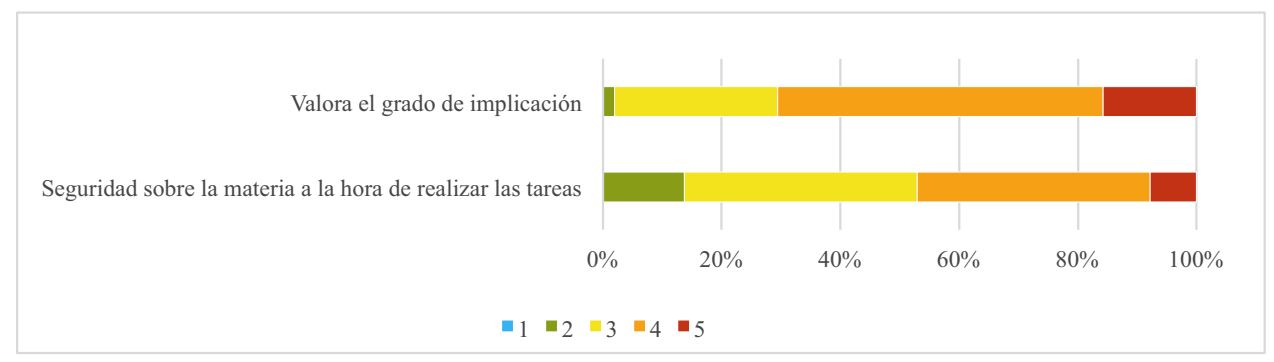

Bloque 2: valoración de la asignatura

La valoración general de la asignatura ha sido positiva (véase Fig. 3). Un 54.9\% la ha valorado con 4 puntos y un $31.4 \%$ con 5 puntos. La mayoría de los ítems, como objetivos, contenidos, metodología, tipo de ejercicios y recursos, han obtenido buena puntuación. En la temporalización (pregunta 11) un $43.2 \%$ la ha puntuado con 2 y 3 puntos. 
Figura 3. Valoración de la asignatura

01. ¿Los objetivos de la asignatura han sido claros desde el principio?

02. ¿Los contenidos han sido actuales, organizados, útiles, interesantes y suficientes?

03. ¿La metodología de enseñanza-aprendizaje en general ha sido adecuada?

04. Valora la utilidad de Codecademy/Khanacademy

05. ¿Te ha resultado difícil realizar los ejercicios de Codecademy/Khanacademy solo/a en casa?

06. ¿Te parece útil el método de clase invertida?

07. ¿Los ejercicios planteados han sido dinámicos y útiles para el aprendizaje?

08. ¿Los ejercicios han tenido un evolución adecuada y adaptada al ritmo del aprendizaje?

09. ¿Te ha resultado difícil enfrentarte al proyecto web?

10. Valora la utilidad del material didáctico proporcionado

11.¿La temporalización de los contenidos ha sido adecuada?

12 ¿El sistema de evaluación es adecuado?

13. Valora la utilidad de Google Classroom

14. ¿Las instalaciones del aula son satisfactorias?

15. Valoración global de la asignatura
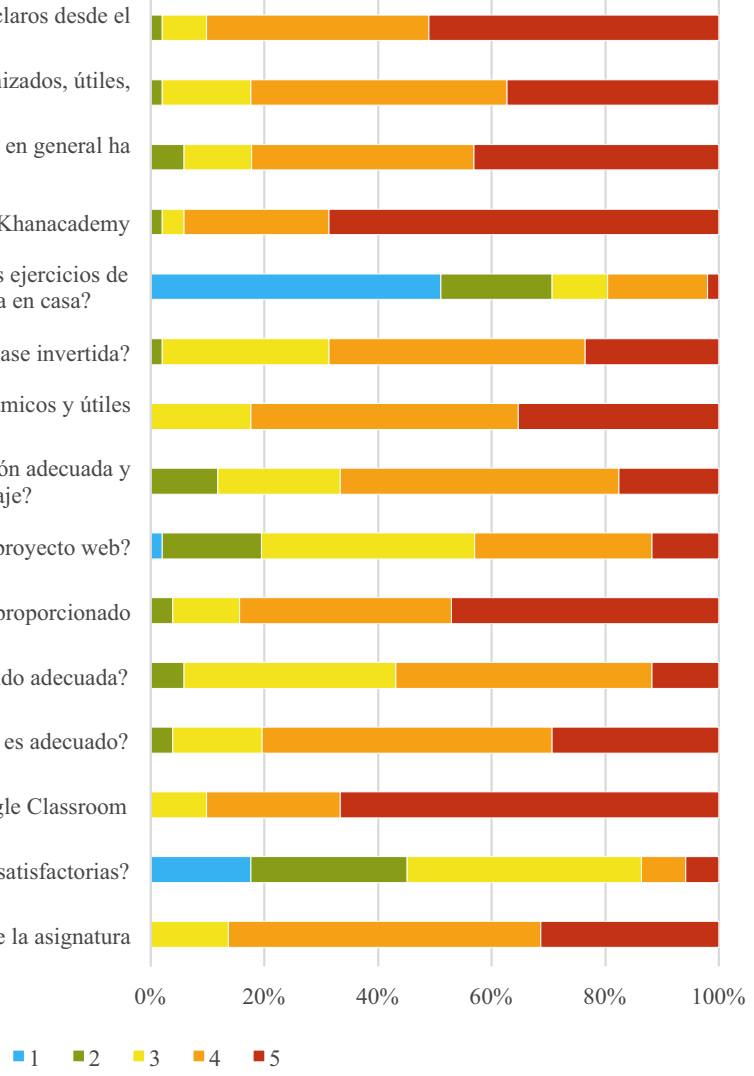

A la pregunta ¿cuánto tiempo aumentarías o disminuirías las horas dedicadas a la clase invertida? el 80.4\% opina que las dejaría tal cual se ha aplicado (véase Fig. 4).

Figura 4. Valoración horas dedicadas a la clase invertida

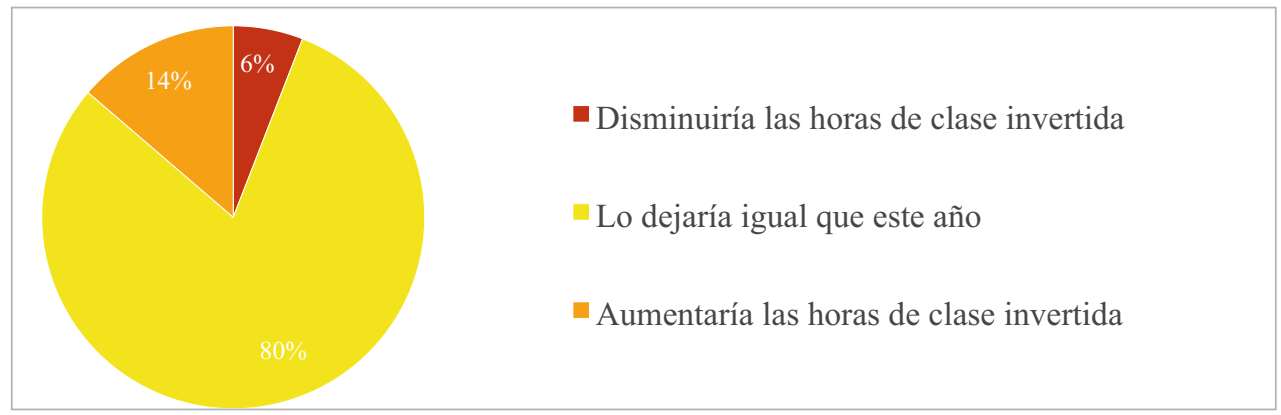


Bloque 3: Opinión y sugerencias del alumnado

Este bloque estaba formado por 4 preguntas abiertas:

- Lo mejor de la asignatura ha sido...

- Lo peor de la asignatura ha sido...

- ¿Qué cosas mejorarías de la asignatura?

- Cualquier otra información que consideres importante.

Las respuestas se han codificado y agrupado por ideas y nos informa de los estudiantes que han querido destacar un tema concreto. A modo de ejemplo, se han extraído frases reales de las contestaciones.

- Dificultad: el $23.1 \%$ piensa que es una asignatura muy compleja y demasiado técnica, p. ej. "es complicada y si pierdes el hilo es difícil retomarlo".

- Contenido: el $65.1 \%$ ha transmitido su satisfacción al conseguir hacer una web desde cero con HTML y CSS. Además, los conocimientos adquiridos los consideran muy útiles para un futuro trabajo. Al $27.3 \%$ les gustaría seguir aprendiendo sobre la materia, p. ej. "aprender a crear webs me parece de las cosas más útiles que hemos hecho".

- Organización, dinámica de clase y explicaciones: el 34.9\% opina que lo mejor ha sido la variedad de estrategias y recursos y su ordenación, p. ej. "la variedad de métodos didácticos". No obstante, el $61.6 \%$ opina que hay poco tiempo para tanto contenido, p. ej. "poco tiempo para explicar lo que la programación puede dar".

- Recursos: al 27.3\% le ha gustado mucho la actividad de cHTeMeLe, y los recursos Khanacademy y Codecademy, p. ej. "muy útil el juego de cartas de HTML". El 7.1\% opina que podría "mejorar la organización y especificación de los links en Classroom".

- Ejercicios: el 14.3\% preferiría hacer más ejercicios prácticos de cada concepto antes del proyecto final, p. ej. "más ejercicios antes del proyecto, es cuando asimilas de verdad".

\subsection{Asistencia}

Los rangos utilizados en el apartado de asistencia van de 10 en 10 puntos (véase Fig. 5). La guía docente de la asignatura establece que el estudiante debe asistir como mínimo al 70\% de las clases para poder acogerse a la evaluación continua. Un alumno que no cumple este porcentaje va a la evaluación final. Aunque desde el principio la asistencia era óptima, p. ej. en el curso 12-13 el 56\% de estudiantes tenían una asistencia mínima del 70\%; ha ido mejorando, p. ej. en el curso $16-17$ el $88.5 \%$ del alumnado asistió a más del $70 \%$ de las clases. 
Figura 5. Asistencia

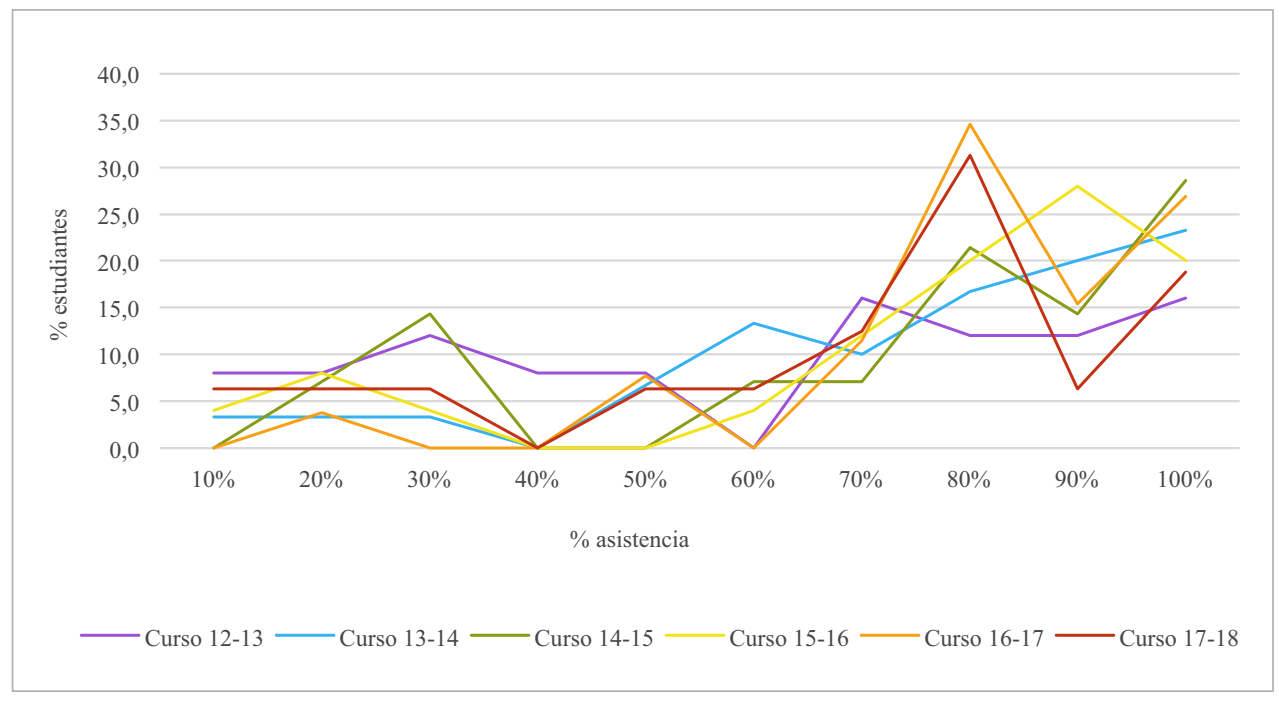

\subsection{Abandonos}

El número de abandonos fue $40 \%$ en el curso $12-13,3.3 \%$ en el $13-14,28.6 \%$ en el $14-15,0 \%$ en $15-16,7.7 \%$ en el $16-17$ y $12.5 \%$ en el curso $17-18$. Aunque la tasa de abandono no ha descendido gradualmente a lo largo de la experiencia, con la nueva propuesta ha disminuido cada curso por debajo de la tasa promedio de la especialidad que es $\mathbf{1 4 . 5 \%}$. Dada la desvinculación de los estudiantes que abandonan el centro, es complejo saber las causas que les han llevado tomar esta decisión. Además, el alumnado de estos estudios son adultos, a veces con cargas familiares y/o en muchos casos trabajando al mismo tiempo, por lo que el abandono en muchas ocasiones es debido a causas ajenas a la asignatura.

\subsection{Calificaciones}

En cuanto a las calificaciones, estas van del 1 al 10 donde el 5 es aprobado. En la gráfica se describen las calificaciones con rangos de 1 punto para simplificarla. A partir del curso 15-16, el número de suspensos es imperceptible y las calificaciones han mejorado (véase Fig. 6). Por ejemplo, el porcentaje de estudiantes que ha logrado más de 7 puntos ha ascendido de un promedio de $59,9 \%$-en la propuesta previa- a un $80,5 \%$. Y sobre todo destacar el $40 \%$ de estudiantes que obtuvieron 9 en el curso $15-16$ o el $46.2 \%$ que consiguieron 8 en el $16-17$. 
Figura 6. Calificaciones

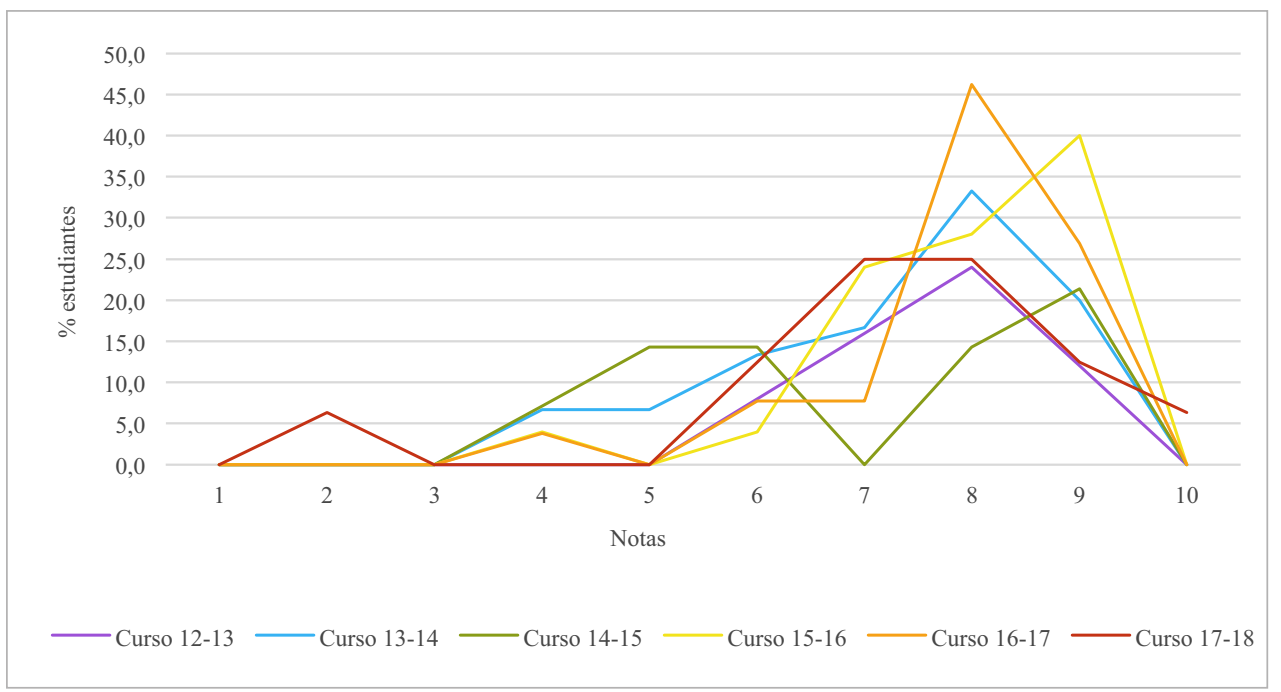

\section{DisCuSión y CONCLUSIONES}

El momento actual nos exige experiencias innovadoras con respecto al proceso de enseñanza-aprendizaje y el uso de las tecnologías de la información y la comunicación (Lázaro Cayuso, 2017). En este estudio, a partir del análisis de una experiencia previa, se ha diseñado una propuesta didáctica nueva. En la tabla 2 se pueden observar las características de la presente propuesta y la realizada anteriormente.

Tabla 2. Características de la propuesta didáctica previa y la experiencia docente presentada en este artículo

\begin{tabular}{|c|c|c|c|c|}
\hline Propuestas & Curso & $\begin{array}{l}\text { Componentes } \\
\text { didácticos }\end{array}$ & $\begin{array}{l}\text { Técnicas e instrumentos / } \\
\text { indicadores }\end{array}$ & Resultados \\
\hline \multirow{3}{*}{$\begin{array}{l}\text { Propuesta } \\
\text { didáctica } \\
\text { previa } \\
\text { (Torres Blasco, } \\
\text { 2016) }\end{array}$} & $12-13$ & \multirow{3}{*}{$\begin{array}{l}\text { - Modelo } \\
\text { pedagógico } \\
\text { expositivo } \\
\text { demostrativo } \\
\text { - ABPr } \\
\text { - ABP }\end{array}$} & \multirow{3}{*}{$\begin{array}{l}\text { - Cuestionario inicial } \\
\text { (perfil alumnado y nivel } \\
\text { de conocimientos) } \\
\text { - Observaciones de aula } \\
\text { - Entrevistas en grupo } \\
\text { (satisfacción estudiantes) } \\
\text { - Calificaciones } \\
\text { - Asistencia } \\
\text { - Tasa de abandono }\end{array}$} & \multirow{3}{*}{\begin{tabular}{|l} 
- Gran complejidad \\
de la asignatura \\
- Excesivas clases \\
teóricas \\
- Inseguridad y \\
desmotivación \\
- Alta tasa de \\
abandono \\
- Buena asistencia \\
- Calificaciones \\
mejorables
\end{tabular}} \\
\hline & $13-14$ & & & \\
\hline & $14-15$ & & & \\
\hline
\end{tabular}




\begin{tabular}{|c|c|c|c|c|}
\hline Propuestas & Curso & $\begin{array}{l}\text { Componentes } \\
\text { didácticos }\end{array}$ & $\begin{array}{c}\text { Técnicas e instrumentos / } \\
\text { indicadores }\end{array}$ & Resultados \\
\hline \multirow{3}{*}{$\begin{array}{l}\text { Nueva } \\
\text { propuesta } \\
\text { didáctica } \\
\text { (ciclo 1) }\end{array}$} & $15-16$ & \multirow{3}{*}{$\begin{array}{l}\text { - Modelo } \\
\text { pedagógico } \\
\text { expositivo } \\
\text { demostrativo } \\
\text { - ABPr } \\
\text { - ABP } \\
\text { - Clase } \\
\text { invertida } \\
\text { - TE (REA) } \\
\text { - Juego serio } \\
\text { gamificado } \\
\text { - Entorno } \\
\text { virtual de } \\
\text { gestión de la } \\
\text { asignatura }\end{array}$} & \multirow{3}{*}{$\begin{array}{l}\text { - Cuestionario inicial } \\
\text { (perfil alumnado y nivel } \\
\text { de conocimientos) } \\
\text { - Observaciones de aula } \\
\text { - Cuestionario de } \\
\text { satisfacción } \\
\text { - Calificaciones } \\
\text { - Asistencia } \\
\text { - Tasa de abandono }\end{array}$} & \multirow{3}{*}{$\begin{array}{l}\text { - Menor tasa de } \\
\text { abandono } \\
\text { - Mayor asistencia } \\
\text { - Mejores } \\
\text { calificaciones } \\
\text { - Mayor } \\
\text { satisfacción del } \\
\text { alumnado } \\
\text { - Facilitación de } \\
\text { adquisición de } \\
\text { competencias }\end{array}$} \\
\hline & $16-17$ & & & \\
\hline & $17-18$ & & & \\
\hline
\end{tabular}

Los logros conseguidos una vez implementada la propuesta han sido:

- Captar la atención con estrategias variadas y dinámicas como el juego cHTeMeLe.

- Realizar un acompañamiento de calidad por parte de la profesora.

- Fomentar la colaboración en la resolución de problemas.

- Hacer partícipe al alumnado de su aprendizaje.

- Promover el trabajo autónomo con la clase invertida.

- Adaptar la asignatura a los diferentes ritmos de aprendizaje con Codecademy y Khanacademy.

- Disminuir las inseguridades y frustraciones con un aprendizaje progresivo.

- Aumentar la motivación y el interés por la materia.

- Potenciar el aprendizaje significativo con el ABPr y ABP.

- Adquirir las competencias para el desarrollo web con HTML y CSS.

En cuanto a los resultados de abandono, asistencia y calificaciones, por una parte se observa que la tasa de abandono aumenta o disminuye según el porcentaje de asistencia, y por otra parte, que los suspensos se reducen y las calificaciones mejoran respecto al estudio anterior.

Por todo ello, se puede verificar que con el cambio pedagógico ha mejorado la propuesta de enseñanza-aprendizaje de la asignatura, y por lo tanto, el desarrollo de las competencias relacionadas con el uso de HTML y CSS creando un aprendizaje activo y significativo. No obstante, uno de los factores importantes para el buen funcionamiento de la asignatura es la asistencia e implicación del alumnado, debido al carácter técnico y alejado del perfil de estos estudios. De esta forma, el alumnado se aprovecha del impacto de los cambios didácticos realizados en la nueva propuesta didáctica. A lo largo de la asignatura, la mayoría de los estudiantes cada vez están más seguros y preparados para empezar a desenvolverse en el mundo laboral, y tie- 
nen recursos para ampliar conocimientos de forma autónoma.

El resultado ha sido una propuesta didáctica de integración metodológica, como el caso de la experiencia de Lázaro Cayuso (2017), donde el ABPr es la base de unión de diferentes componentes como la clase invertida y el trabajo colaborativo; o el de la experiencia de Cáceres Taladriz (2016), que implanta una propuesta de hasta 7 componentes, entre los que está incluida la clase invertida, la gamificación, el trabajo colaborativo y la instrucción por pares. Este último autor afirma que la combinación adecuada de estas metodologías permite mantener la atención y el interés del alumnado. En nuestro caso, el ABPr se propone como pilar de esta nueva propuesta, por ser un método constructivista donde se aprende haciendo. No obstante, se hace énfasis en que el resto de componentes son imprescindibles para conseguir el éxito. Es decir, no se podría empezar directamente con un ABPr porque los estudiantes no tienen conocimientos previos. Por lo tanto, el juego serio gamificado cHTeMeLe, el ABP y la clase invertida -con la ayuda de los REA Codecademy o Khanacademy- han contribuido a crear el contexto adecuado para que el ABPr funcione. Concretamente, la clase invertida y los REA son los que han facilitado ese tiempo valioso en clase para la resolución de problemas y el desarrollo del proyecto. De esta manera, la profesora deja de ser una mera transmisora de conocimiento para convertirse en facilitadora.

Sin embargo, a algunos estudiantes le sigue pareciendo una asignatura compleja y todos opinan que requiere excesiva dedicación. Así pues, de cara a futuros cursos se está pensando en la posibilidad de reducir el volumen de contenidos obligatorios - para que los estudiantes dispongan de más tiempo para obtener una base más sólida- y dejar materiales opcionales de ampliación. Además, se propone explorar la instrucción por pares que trabaja problemas por parejas (Cáceres Taladriz, 2016) y el aprendizaje en proyectos grupales similares a una experiencia profesional. De esta forma, se trabajaría la gestión del proyecto y donde sería adecuada la inclusión de métodos ágiles como Scrum. Las metodologías ágiles utilizadas en el desarrollo de productos multimedia están aplicándose al mundo de la educación con gran éxito, ya que desarrollan capacidades, habilidades y aptitudes que les serán de utilidad en el entorno académico y luego en su carrera profesional (Kuz et al., 2018). Gracias a estas metodologías ágiles se prevé que aumentará la participación de los estudiantes, fomentará el trabajo en equipo, la autoorganización, el autoaprendizaje y la autoevaluación, que en definitiva, enriquecen las capacidades de crecimiento personal.

\section{REFERENCIAS BIBLIOGRÁFICAS}

Alexander, B., Ashford-Rowe, K., Barajas-Murphy, N., Dobbin, G., Knott, J., Mccormack, M., Pomerantz, J., Seilhamer, R. y Weber, N. (2019). Horizon Report. 2019 Higher Education Edition. https://library.educause.edu/resources/2019/4/2019horizon-report

Amiri, F. (2011). Programming as design: The role of programming in interactive media curriculum in art and design. International Journal of Art \& Design Education, 30(2), 200-210. https://doi.org/10.1111/j.1476-8070.2011.01680.x

Botella Nicolás, A. M. y Ramos Ramos, P. (2019). La teoría de la autodeterminación: un marco motivacional para el aprendizaje basado en proyectos. Contextos Educativos. Revista de Educación, 24, 253-269. https:/doi.org/10.18172/con.3576 
Cáceres Taladriz, C. (2016). Evolución de la clase magistral a la clase invertida: flipped classroom no basta. En M. Rubio Sánchez, J. Urquiza Fuentes, y C. Cáceres Taladriz (Eds.), VII Jornadas en Innovación y TIC Educativas. JITICE 2016 (pp. 41 44). Universidad Rey Juan Carlos Servicio de Publicaciones. https://eciencia.urjc. es/handle/10115/14827

Campaña, J. R., Marín, A. E., Ros, M., Sánchez, D., Medina, J. M., Vila, M. A., Ruiz, M. D., Cuéllar, M. P. y Martin-Bautista, M. J. (2016). Metodologías activas y gamificación en las asignaturas de iniciación a la programación. En M. Torres Gil y J. J. Cañadas Martínez (Eds.), XXII Jornadas sobre Enseñanza Universitaria de la Informática (pp. 241-248). Editorial Universidad de Almería. http://www2.ual.es/ jenui2016/files/actas_jenui2016.pdf

De Benito Crosetti, B. y Salinas Ibáñez, J. M. (2016). La Investigación Basada en Diseño en Tecnología Educativa. RIITE. Revista Interuniversitaria de Investigación en Tecnología Educativa, 0, 44-59. https://doi.org/10.6018/riite2016/260631

Dorn, B. y Guzdial, M. (2010). Learning on the job: Characterizing the programming knowledge and learning strategies of web designers. $\mathrm{CHI}$ '10: 28th International Conference on Human Factors in Computing Systems, 2, 703-712. https://doi. org/10.1145/1753326.1753430

Gallego-Durán, F. J., Villagrá-Arnedo, C. J., Satorre Cuerda, R., Compañ, P., MolinaCarmona, R. y Llorens Largo, F. (2014). Panorámica: serious games, gamification y mucho más. ReVision, 7(2), 13-23. http://hdl.handle.net/10045/37972

Gallego Carrillo, M., Gortázar Bellas, F. y Montalvo Herranz, S. (2016). El Aprendizaje Basado en Proyectos y la Clase Invertida para acercar el mundo real al aula. En M. Rubio Sánchez, J. Urquiza Fuentes y C. Cáceres Taladriz (Eds.), VII Jornadas en Innovación y TIC Educativas. JITICE 2016 (pp. 45-48). Universidad Rey Juan Carlos Servicio de Publicaciones. https://eciencia.urjc.es/handle/10115/14827

Gutiérrez Quintana, E. (2007). Técnicas e instrumentos de observación de clases y su aplicación en el desarrollo de proyectos de investigación reflexiva en el aula y de autoevaluación del proceso docente. En S. Pastor Cesteros y S. Roca Marín (Eds.), XVIII Congreso Internacional de la ASELE (pp. 336-342). Servicio de Publicaciones de la Universidad de Alicante. https://eciencia.urjc.es/handle/10115/14827

Imaz, J. I. (2015). Aprendizaje Basado en Proyectos en los grados de Pedagogía y Educación Social: "¿Cómo ha cambiado tu ciudad?" Revista Complutense de Educación, 26(3), 679-696. https://doi.org/10.5209/rev_RCED.2015.v26.n3.44665

Kuz, A., Falco, M. y Giandini, R. S. (2018). Comprendiendo la Aplicabilidad de Scrum en el Aula: Herramientas y Ejemplos. Revista Iberoamericana de Tecnología en Educación y Educación en Tecnología, 21, 62-70. https://doi. org/10.24215/18509959.21.e07

Lázaro Cayuso, P. (2017). Innovaciones metodológicas para la sociedad digital: aprendizaje basado en proyectos, aprendizaje colaborativo, flipped classroom e inteligencias múltiples. Tendencias pedagógicas, 30, 339-354. https://dialnet. unirioja.es/servlet/articulo?codigo $=6164804$

Luján Mora, S. y Saquete Boró, E. (2017). Mejora en el aprendizaje a través de la combinación de la clase invertida y la gamificación. En A. Gómez Mancha y R. Rodríguez-Echevarría (Eds.), JENUI 2017: XXIII Jornadas sobre Enseñanza Univer- 
sitaria de la Informática (pp. 213-220). Asociación de Enseñantes Universitarios de la Informática. https://jenui2017.unex.es/actas_jenui2017.pdf

Matas, A. (2018). Diseño del formato de escalas tipo Likert: Un estado de la cuestión. Revista Electronica de Investigacion Educativa, 20(1), 38-47. https://doi. org/10.24320/redie.2018.20.1.1347

Olsson, M. y Mozelius, P. (2016). On design of online learning environments for programming education. En J. Novotna y A. Jancarik (Eds.), 15th European Conference on e-Learning, ECEL (pp. 533-539). Academic Conferences and Publishing International Ltd. https://www.researchgate.net/publication/309566050_On_design_of_online_learning_environments_for_programming_education

Sánchez Rodríguez, J., Ruiz Palmero, J. y Sánchez Vega, E. (2014). Las clases invertidas: beneficios y estrategias para su puesta en práctica en la educación superior. En M. C. Domínguez Garrido, M. L. Cacheiro González y J. Dulac Ibergallartu (Eds.), XIX Congreso Internacional de Tecnologías para la Educación y el Conocimiento - VI Congreso Pizarra Digital: Diversidad, Estrategias y Tecnologías. Diálogo entre culturas (pp. 1-11). UNED. https://riuma.uma.es/xmlui/handle/10630/7821

Sosa Sánchez-Cortés, R., García Manso, A., Sánchez Allende, J., Moreno Díaz, P. y Reinoso Peinado, A. J. (2005). B-Learning y Teoría del Aprendizaje Constructivista en las Disciplinas Informáticas: Un esquema de ejemplo a aplicar. En B. González Pereira (Ed.), Recent research developments in learning technologies. III International Conference on Multimedia and Information \& Communication Technologies in Education (pp. 1-6). Formatex. https://www.academia. edu/10154771/B-Learning_y_Teoría_del_Aprendizaje_Constructivista_en_las_ Disciplinas_Informáticas_Un_esquema_de_ejemplo_a_aplicar

Torres Blasco, C. (2016). Metodología de enseñanza-aprendizaje de los lenguajes informáticos HTML y CSS para estudiantes con predominancia lateral derecha: El caso de estudiantes de Diseño Gráfico. Universitat Oberta de Catalunya. http:// hdl.handle.net/10609/53644

Universidad Pública de Navarra. (s. f.). Cuestionario de satisfacción del estudiante con la docencia. http://www.unavarra.es/serviciocalidadyorganizacion/calidadde-los-titulos/mapa-de-procesos/todos/soporte/satisfaccion-de-los-estudiantescon-la-docencia

Universitat Autònoma de Barcelona. (s. f.). Encuesta de evaluación y satisfacción. https://www.uab.cat/web/estudiar/calidad-docente/encuestas-de-evaluacion-ysatisfaccion-1345738816964.html

Venter, M. (2020). Gamification in STEM programming courses: State of the art. En A. Cardoso, G. R. Alves y T. Restivo (Eds.), 2020 IEEE Global Engineering Education Conference (EDUCON) (pp. 859-866). IEEE Computer Society. https://doi. org/10.1109/EDUCON45650.2020.9125395 